\title{
Editorial
}

\section{eCAM: Proteomics, BioArt and Integrative Medicine?}

\author{
Edwin L. Cooper \\ Laboratory of Comparative Neuroimmunology, Department of Neurobiology, David Geffen School of Medicine \\ at UCLA, University of California, Los Angeles, CA 90095-1763, USA
}

For 2010, we now begin the 10th year of the 21st century and $e C A M$ has occupied five of them. $e C A M$ covers have presented art to focus on some of the cultures, where integrative medicine was born and still thrives side by side with western medicine. These have been represented by art from Japan, Korea, India, Italy, China. Other vibrant cultures where CAM flourishes were not intentionally omitted, merely due to problems of space and necessary, sometimes complicated, paperwork associated with borrowing from museums. Here are descriptions of covers that represent art and culture spanning several millennia and those that were devoted to origins of CAM and integrative medicine.

Volumes 1 and 2-Japan (2004-05): The woodblock print by Hiroshige of a traveler on a bridge reflects our hope that CAM will bridge cultures as well as CAM and modern biomedicine. There is no quick indication of a connection to Kampo that surely existed during that period. Volumes 3 and 4-Korea (2006-07): A Korean screen (artist unknown from the 18th century Cho-San Dynasty), pictures 12 representations of long life, including sun, moon and clouds, mountain, bamboo, pint tree, rocks, turtles, cranes, deer, water and mushroom (Ganodermae). This cover symbolizes the strong connection in Korean culture between nature and healing; already, the inclusions are sources of products used in ancient medicines. Volumes 5 and 6-India (2008-09): Keeping a focus on Ayurveda, this cover is entitled: Tree Worship (India, Rajasthan, ca. 1730-50, whose artist is unknown). Like the two previous covers from Korea and Japan, there is a fair amount of symbolism relevant to CAM.

For reprints and all correspondence: Edwin L. Cooper, Laboratory of Comparative Neuroimmunology, Department of Neurobiology,

David Geffen School of Medicine at UCLA, University of California, Los Angeles, CA 90095-1763, USA. Tel: +1-310-825-9567;

Fax: +1-310-825-2224; E-mail: ecam@mednet.ucla.edu
Supplement 4-Europe (2007): ETM 2007 European Traditional Medicine International Congress, Vinci, Italy, October 4-6, 2007. 'Star of Bethlehem: (Family Liliaceae, Ornithogalum umbellatum L.)', Leonardo da Vinci, Italy; pen and ink over chalk on paper. Reproduced with kind permission from the Royal Collection 2006, Her Majesty Queen Elizabeth II. The concept of nature is fundamental in Leonardo's art. The Star of Bethlehem is an ornamental plant; its florescence can bear 20 flowers. A toxic plant and poisonous if ingested, yet its parts contain cardiac glycosides with the bulbs containing a higher percentage of toxin. Preliminary analyses (1970s) were devoted to its possible treatment of heart failure but were abandoned. Supplement 6-Korea (2009): Sasang Constitutional Medicine: as a holistic tailored medicine. The Banquet of Seowangmo (Xiwangmu), Queen Mother of the West, Joseon dynasty (1392-1910), 18th-19th century Screen/scroll, painting, Eight-panel screen, ink, color and gold on silk.

These ancient cultures now tend to dispel references to that which is 'art', appealing to the senses and not proven through the rigors of exact science. Closer to us, eCAM was founded with the intention of adding scientific definitiveness, precision and subsequent reproducibility centered on evidence-based analyses. Thus, we have two representations: ancient art and a beginning exodus from ancient biomedicine. Now we come to Volume 7 and again a chance to make two culture statements concerning aspects of contemporary art that with some imagination and introspection may reflect the refinement of evidence-based CAM; after all art if viewed now is everywhere, even in the products of test tubes and Petri dishes! Volume 7 (2010) represents a transition from ancient medicine and ancient medical practice to $e C A M$ and 21 st century contemporary art (BioArt). We begin volume 7 with a figure taken from the paper by a 
group in Japan. The very key words confirm that they use cutting edge technology to elucidate features of 'Oketsu', a concept in Kampo [e.g. protein chip surface-enhanced laser desorption/ionization time-of-flight mass spectrometry (SELDI-TOF MS) keishibukuryogan (KBG)]. There is actually a place for contemporary art that parallels closely with what is visualized in the current cover and referred to as BioArt.

The 21 st century integrative medicine and its visual results can connect with BioArt. Matsumoto et al. (1) have analyzed 'Oketsu' a pathophysiologic concept in Japanese traditional (Kampo) medicine, primarily denoting blood stasis/stagnant syndrome. They explored plasma protein biomarkers and/or diagnostic algorithms for 'Oketsu' (1). Sixteen rheumatoid arthritis patients were treated with KBG, a Kampo medicine that improves 'Oketsu'. SELDI protein profiles gave 266 protein peaks; expression was significantly different between the 'Oketsu' and 'non-Oketsu' states. Hierarchical clustering gave three major clusters (I, II, III), and construction of the decision tree suggested the possible development of a diagnostic algorithm for 'Oketsu'. This concept of Kampo medicine has a physical basis in terms of blood-protein profiles. Perhaps we may establish objective criteria for diagnosing 'Oketsu' using a combination of proteomic and bioinformatics-based classifications. To assert their concern for good science, the authors agree: 'Therefore, it is very important to standardize the diagnostic criteria for "Oketsu" objectively by using a universally acceptable scientific method' $(2,3)$. A similar recognition will center on more similar contemporary CAM art in a future publication (4).

Now we connect art that connects to CAM or integrative medicine. We borrowed figure 4 from the above paper and reproduced its legend here. It has vibrant colors of reds and greens, and has been tested on lay readers of $e C A M$ and others chosen randomly. Invariably, the response was something like:-'it is representative of modern/contemporary art! My hunch was right! This is a hierarchical clustering of 55 SELDI profiles. Clustering was based on the 266 differential peaks obtained from 55 SELDI profiles and Oketsu/non. Oketsu classification. The red (peak maximum $=1$ ) or green (peak minimum $=0$ ) color indicates the relative intensity, i.e. higher than or lower than the median value (black color).

BioArt of the 21st century exists everywhere. Finding art in nature is hardly a new concept in general and in biology in particular. At every level of biological organization, colors abound, shapes are numerous and there is symmetry and asymmetry yin and yang! Contemporary art is multifaceted, expansive and often expressive. However, one of its divisions stands out and is most appropriate. BioArt interprets living matter and resulting works of art are produced in laboratories and/or artists' studios. Biotechnology is one tool and it includes genetic engineering, tissue culture; it is mostly restricted to 'living forms'. Of course, debate can sometimes center around when can matter be considered as alive or living? Bioartists use cells, proteins and living tissue. 'BioArt' was coined by Eduardo Kac (5) in relation to his artwork 'Time Capsule'. It originated in the late 1900s through the art of pioneers $(5,6)$. BioArt began to be more widely practiced about in the beginning of the 21 st century (2005). Thus, it may be considered first among 21st century contemporary art. Genesis, writes Kac, is a transgenic artwork that explores the intricate relationship between biology, belief systems, information technology, dialogical interaction, ethics, and the Internet. Above all, it is a work that forces us to examine the creative and destructive power of humans - a power we inherited along with words and numbers'.

Integrated genomic analyses examine the genomes of organisms, their hereditary information encoded in DNA. The field includes intensive efforts to determine the entire DNA sequence of organisms and to fine-scale genetic mapping efforts. With this in mind, clearly CAM strategies should be devoted to zeroing in or pinpointing targets where effects might be operative and measured. Understanding the molecular mechanisms of disease requires an introduction of molecular diagnostics into medical practice (3). Current medicine employs elements of molecular diagnostics and is usually on single genes. Medicine in the post-genomic era will utilize thousands of molecular markers associated with disease that are provided by high-throughput sequencing and functional genomic, proteomic and metabolomic analyses and thus BioArt will emerge!

\section{References}

1. Matsumoto C, Kojima T, Ogawa K, Kamegai S, Oyama T, Shibagaki Y, et al. A proteomic approach for the diagnosis of 'Oketsu' (blood stasis), a pathophysiologic concept of Japanese traditional (Kampo) medicine. Evid Based Complement Alternat Med 2008;5:463-74.

2. Cooper EL. Complementary and alternative medicine, when rigorous can be science. Evid Based Complement Alternat Med 2004;1:1-4.

3. Cooper EL. eCAM: integrative genomics and fecundity. Evid Based Complement Alternat Med 2009;6:129-31.

4. Kim SK, Kim J, Ko E, Kim H, Hwang D-S, Lee S, et al. Gene expression profile of the hypothalamus in DNP-KLH immunized mice following electroacupuncture stimulation. 2010, in press.

5. Kac E. Telepresence \& Bio art: Networking Humans, Rabbits and Robots. Ann Arbor: University of Michigan Press, 2005, 250-1.

6. Gessert G. Art and Genetics Bibliography. Leonardo On-Line: Art, Science and Technology, 1996 (Updated January 2005) http:// www.leonardo.info/isast/spec.projects/art + biobiblio.html (Accessed on December 2009). 


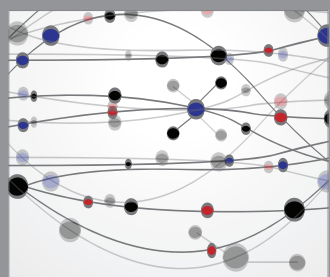

The Scientific World Journal
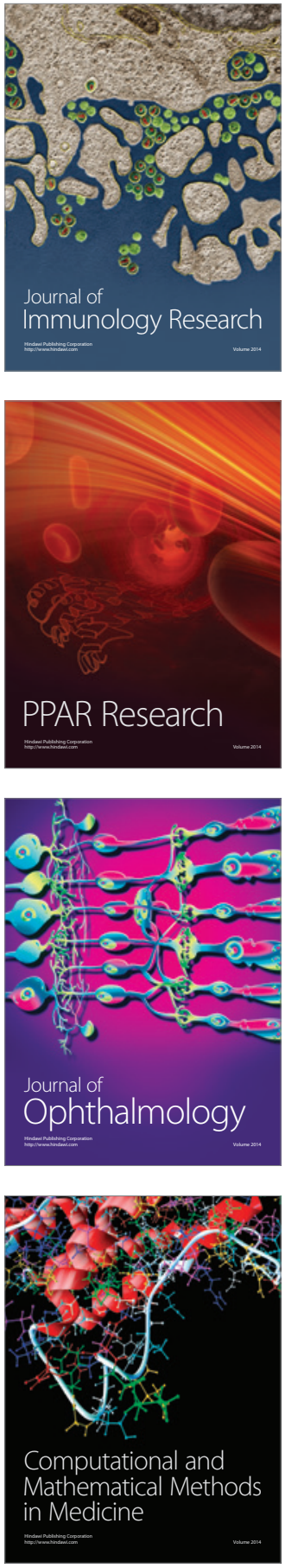

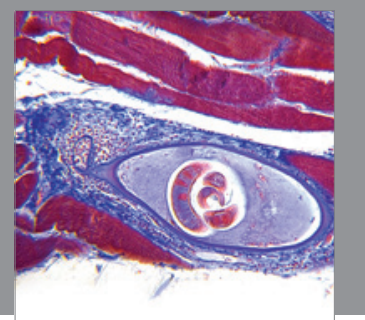

Gastroenterology

Research and Practice
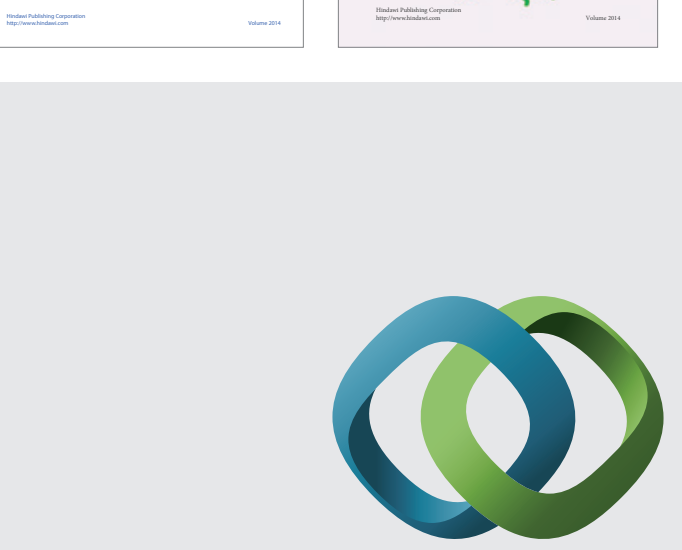

\section{Hindawi}

Submit your manuscripts at

http://www.hindawi.com
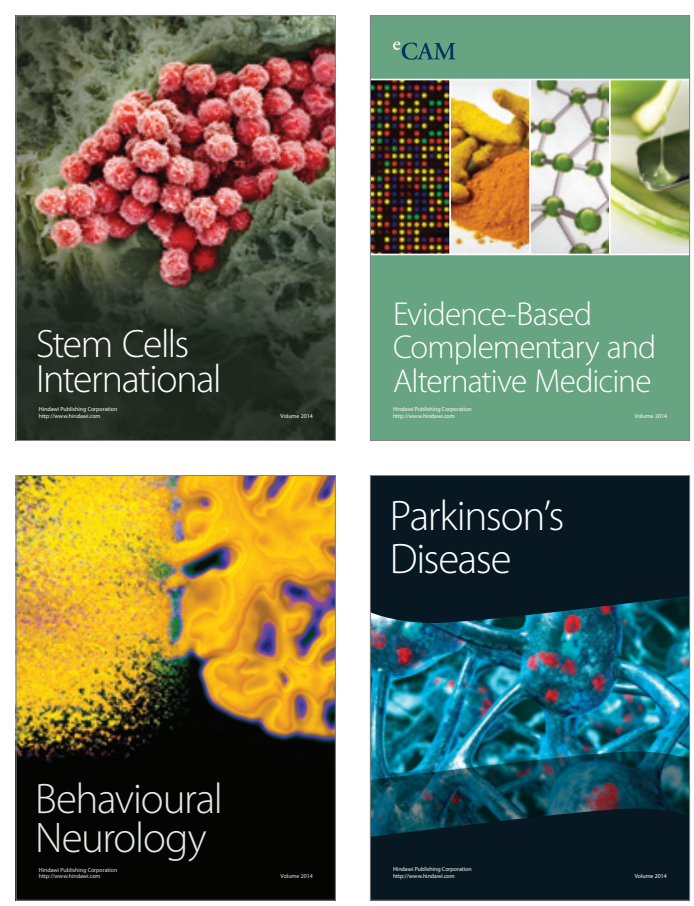

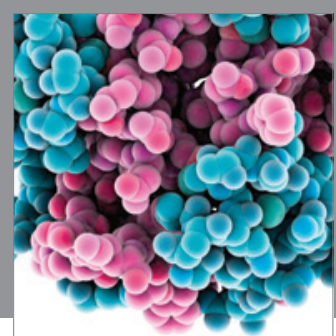

Journal of
Diabetes Research

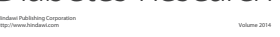

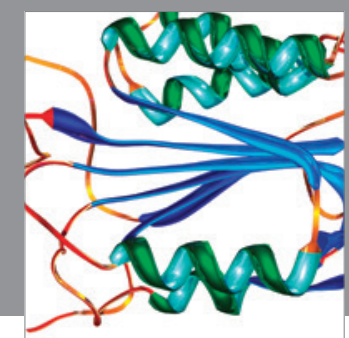

Disease Markers
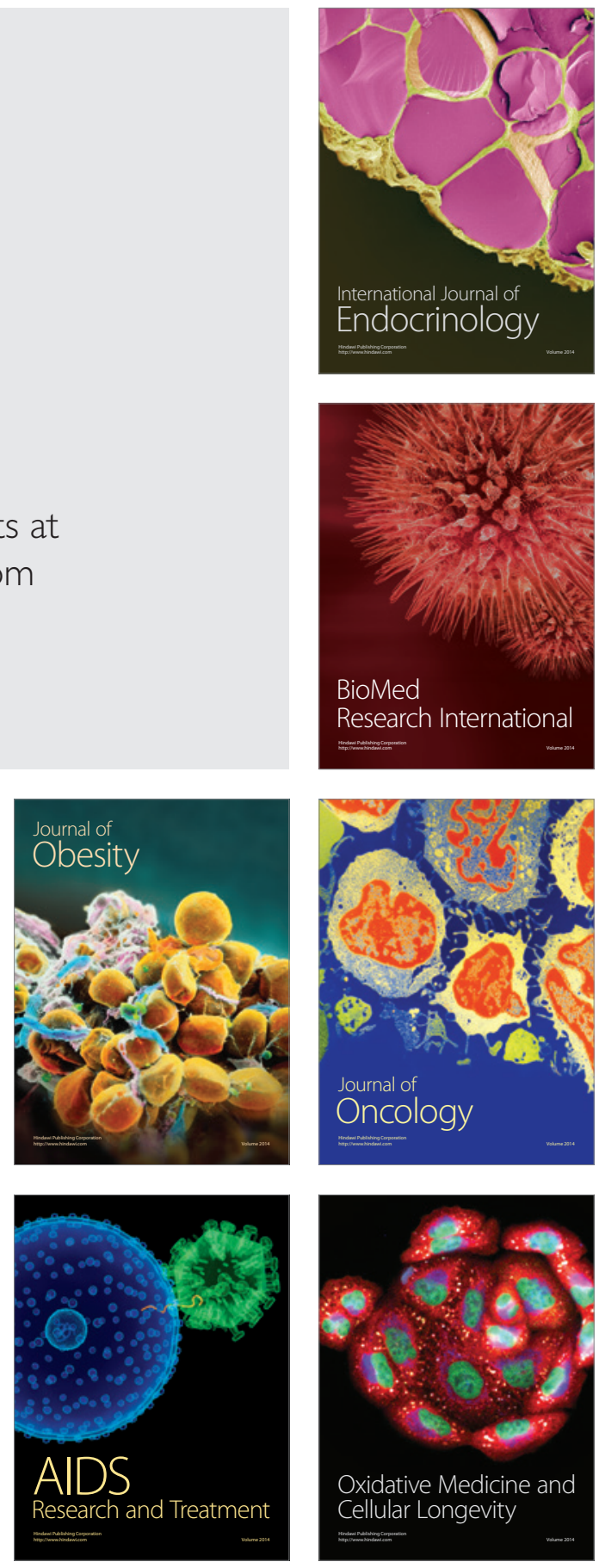